\title{
Prognostic value of soluble ST2 and soluble LR11 on mortality and cardiovascular events in peritoneal dialysis patients
}

Yu Bum Choi ${ }^{1+}$, Mi Jung Lee ${ }^{1,2 \dagger}$, Jung Tak Park ${ }^{3}$, Seung Hyeok Han ${ }^{3}$, Shin-Wook Kang ${ }^{3}$, Tae-Hyun Yoo ${ }^{3}$ and Hyung Jong $\mathrm{Kim}^{1 *}$ (D)

\begin{abstract}
Background: Although the soluble form of suppression of tumorigenicity 2 (SST2) and soluble low-density lipoprotein receptor relative with 11 ligand-binding repeats (sLR11) have emerged as novel cardiovascular biomarkers in patients with cardiovascular disease, their prognostic value has not been fully investigated in peritoneal dialysis (PD) patients.

Methods: We included 74 prevalent PD patients from a prospective cohort and measured serum SST2 and SLR11 concentrations by an enzyme-linked immunosorbent assay. The association of these biomarkers and all-cause mortality and major adverse cardiac and cerebrovascular events (MACCEs) was evaluated.

Results: During a follow-up of 38.5 months, all-cause deaths and MACCEs were observed in 13 (17.6\%) patients and 23 (31.3\%) patients. Multivariable Cox analyses demonstrated that greater sST2 was independently associated with higher risk of all-cause mortality $(\geq 75.8 \mathrm{ng} / \mathrm{mL}$; hazard ratio $[H R]=5.551 ; 95 \%$ confidence interval $[\mathrm{Cl}]=1.360-22.660)$ and MACCEs $(\geq 72.5 \mathrm{ng} / \mathrm{mL} ; \mathrm{HR}=4.609 ; 95 \% \mathrm{Cl}=1.608-13.208)$. Furthermore, sST2 showed additive predictive value for mortality to the base model including traditional risk factors (net reclassification index $=0.598, P=0.04$ ). sLR11 was not significantly associated with all-cause mortality or MACCE.

Conclusions: SST2, but not SLR11, indicated a significant prognostic value for all-cause mortality and cardiovascular events in PD patients. Further research is needed to validate emerging biomarkers in these populations.
\end{abstract}

Keywords: Biomarker, Major adverse cardiac and cerebrovascular events, Peritoneal dialysis, Soluble ST2, Soluble LR11

\section{Background}

Although the survival rate of patients with chronic kidney disease (CKD) has improved, cardiovascular disease remains a major cause of mortality in this population [1]. In CKD, cardiovascular changes are attributed to the kidney disease itself or to dialysis treatment when CKD progresses to end-stage renal disease (ESRD) [1, 2]. Indeed, approximately $20 \%$ or more of patients on dialysis develop

\footnotetext{
* Correspondence: khj04@cha.ac.kr

${ }^{\dagger}$ Yu Bum Choi and Mi Jung Lee contributed equally to this work. 1 Department of Internal Medicine, CHA Bundang Medical Center, CHA University, 59 Yatap-ro, Bundang-gu, Seongnam-si, Korea Full list of author information is available at the end of the article
}

systolic or diastolic dysfunction, suggesting that cardiovascular abnormalities are prevalent in ESRD patients [3]. Therefore, because predicting the risk of cardiovascular disease is expected to play a crucial role in improving the prognosis of cardiovascular disease, several studies have addressed this issue by evaluating new biomarkers.

Suppression of tumorigenicity 2 (ST2) is a member of the interleukin 1 receptor family, and its expression is stimulated by myocardial stress and injury [4]. ST2 has two different forms: a cardio-protective transmembrane receptor (ST2L) and a soluble decoy receptor (sST2) [5-7]. In contrast to ST2L, high concentrations of SST2 are associated with cardiac fibrosis, myocardial hypertrophy and

C C The Author(s). 2020 Open Access This article is licensed under a Creative Commons Attribution 4.0 International License, which permits use, sharing, adaptation, distribution and reproduction in any medium or format, as long as you give appropriate credit to the original author(s) and the source, provide a link to the Creative Commons licence, and indicate if changes were made. The images or other third party material in this article are included in the article's Creative Commons licence, unless indicated otherwise in a credit line to the material. If material is not included in the article's Creative Commons licence and your intended use is not permitted by statutory regulation or exceeds the permitted use, you will need to obtain permission directly from the copyright holder. To view a copy of this licence, visit http://creativecommons.org/licenses/by/4.0/ The Creative Commons Public Domain Dedication waiver (http://creativecommons.org/publicdomain/zero/1.0/) applies to the data made available in this article, unless otherwise stated in a credit line to the data. 
ventricular dysfunction [6-8]. Recent evidence has indicated that sST2 is a useful prognostic marker, especially in congestive heart failure $[9,10]$. Low-density lipoprotein receptor relative with 11 ligand-binding repeats (LR11), a member of the low-density lipoprotein receptor relatives, is highly expressed in vascular smooth muscle cells exhibiting enhanced migration, which leads to atherosclerosis [1115]. In addition to LR11, the soluble form of LR11 (sLR11) also promotes vascular smooth muscle cell proliferation and migration, suggesting that sLR11 levels reflect the degree of vascular smooth muscle cell migration [14-21]. In patients with coronary artery disease [18, 19], familial hypercholesterolemia [21] and type 2 diabetes $[16,20]$, sLR11 levels were found to be increased.

To date, the prognostic value of sST2 and sLR11 has not been investigated in patients undergoing peritoneal dialysis (PD). Only a few studies have evaluated the association between clinical outcomes and SST2 in nondialysis CKD patients and ESRD patients on haemodialysis (HD) or haemodiafiltration [22-25] and there are no studies that have focused on sLR11 in patients with kidney diseases. Therefore, we assessed the association of sST2 and sLR11 with adverse clinical outcomes in PD patients. Moreover, we compared the predictive value of sST2 and sLR11 with high-sensitivity C-reactive protein (hs-CRP) to test their clinical relevance as emerging cardiovascular biomarkers in these populations.

\section{Methods}

\section{Study participants}

This study involved participants from a prospective observational cohort of prevalent PD patients that was designed to investigate factors associated with cardiovascular risk and mortality in the Yonsei University Health System (YUHS). The details of this cohort were reported previously [26]. Among the 102 patients initially enrolled, 5 participants who had insufficient sample volumes for analysis, 7 participants who withdrew before additional blood analysis and 21 participants who declined to store their blood samples were excluded. Finally, 74 participants were analyzed in this study.

\section{Collection of clinical and biochemical data}

Clinical data, including age, sex, cause of ESRD, duration of PD treatment, cardiovascular disease history, smoking status, and medications, were collected at study enrolment by a well-trained study coordinator. Smoking history was collected using a questionnaire. Smoking status was categorized as never-smokers and ever-smokers. Cause of ESRD, duration of PD treatment, comorbid diseases, and medications were collected by careful chart review. Cardiovascular disease was defined as coronary artery disease, peripheral artery disease, or cerebrovascular disease. Coronary artery disease was defined as an acute coronary syndrome or angina requiring coronary artery angioplasty or coronary artery bypass. Peripheral artery disease was defined as ischaemic limb loss and/or ulceration requiring peripheral artery revascularization. Cerebrovascular disease was defined as a transient ischaemic attack, cerebral infarction or carotid endarterectomy. Diabetes mellitus was defined as a history of diagnosis or taking oral hypoglycaemic agents or insulin treatment. Anthropometric measures and blood pressure were determined by a single skilled nurse from our PD unit in the morning after complete overnight dialysate emptying. Height and weight were measured and body mass index was calculated. Blood pressure was measured using a validated automatic oscillometric device. The average value of two measures was recorded. To alleviate the variability from overnight peritoneal dialysate, overnight dialysate was not changed. Overnight fasting blood (12-h) was collected at the time of study enrolment and biochemical variables were measured in the YUHS laboratory. For storing blood samples, an additional $10 \mathrm{~mL}$ of whole blood was collected, and the aliquots of serum and plasma were stored in a deep freezer at $-70{ }^{\circ} \mathrm{C}$. Kt $/ \mathrm{V}$ urea was calculated by PD Adequest 2.0 for Windows software (Baxter Healthcare, Deerfield, IL, USA) for the assessment of dialysis adequacy.

\section{Assessment of sST2, sLR11, and hs-CRP concentrations}

Using stored samples, serum sST2, and sLR11 concentrations were measured by a colourimetric enzymelinked immunosorbent assay (ELISA; sST2, Presage ${ }^{\circ}$ ST2 Assay, Critical Diagnostics, San Diego, CA, USA; sLR11, MBS-167426, MyBioSource, San Diego, CA, USA). Interand intra-assay coefficients of variation were both $<10 \%$ for $\mathrm{sST} 2$, and $<15$, and $<10 \%$, respectively, for sLR 11 . hs-CRP concentrations were measured by a BNII analyser (Dade Behring, Newark, DE, USA) using a latexenhanced immunonephelometric method.

\section{Follow-up and study outcomes}

Patients were followed every 3 months at the PD unit of the YUHS through September 2018. All adverse clinical events, including deaths and hospitalizations, were recorded in the database of our PD unit. The primary outcome was all-cause mortality and the secondary outcome was a major adverse cardiac and cerebrovascular event (MACCE). Death or hospitalization from an acute coronary syndrome, stable angina performing coronary revascularization procedures, newly developed congestive heart failure or cerebrovascular events was defined as MACCE. Patients who did not experience primary outcome were censored at the end of the study date and those who were lost to follow-up were censored at the date of the last examination. If patients 
underwent a kidney allograft or changed dialysis modality to $\mathrm{HD}$, they were censored at the date of the last PD treatment. If death occurred within 2 months after transfer to HD, the death was considered as a death from PD.

\section{Statistical analysis}

Statistical analysis was performed using SPSS for Windows version 20.0 (IBM Corp., Armonk, NY, USA) and $\mathrm{R}$ software ( $\mathrm{R}$ Foundation for Statistical Computing, Vienna, Austria; www.r-project.org). Continuous variables are expressed as the mean \pm standard deviation (SD) or the median (interquartile range) and categorical variables are expressed as the raw number (percentage). To evaluate the association of sST2 and sLR11 with study outcomes, study participants were dichotomized based on the median values of each marker. The cut-off value for sST2 and sLR11 was also calculated from receiver operating characteristic (ROC) analyses with the calculated area under the ROC curve (AUC) in accordance with previous studies [23, 24]. The value showing the highest sensitivity was determined as the cut-off. The calculated cut-off values of sST2 for all-cause mortality and MACCEs were $75.8 \mathrm{ng} / \mathrm{mL}$ (sensitivity, 69.2\%; specificity, $60.7 \%$ ) and $72.5 \mathrm{ng} / \mathrm{mL}$ (sensitivity, 65.2\%; specificity, 58.8\%). The calculated cut-off of sLR11 for all-cause mortality and MACCEs was $14.9 \mathrm{ng} / \mathrm{mL}$ (61.5\% sensitivity and $44.3 \%$ specificity for all-cause mortality; $52.5 \%$ sensitivity and $41.2 \%$ specificity for MACCEs). Cumulative survival curves were constructed using the Kaplan-Meier method and between-group survival was compared using the log-rank test. To determine the independent prognostic value of sST2 and sLR11 for allcause mortality and MACCEs, Cox proportional regression analyses were performed. A multivariable model was constructed with significant variables from univariate analyses: sex, age, diabetes mellitus, and cardiovascular disease. Subsequent multivariable analysis was performed with additional variables that were known to be associated with adverse clinical outcomes in PD patients: duration of PD, smoking status, body mass index, and haemoglobin concentrations. Using a fractional polynomial model, sST2 and sLR11 were also evaluated as continuous variables. To compare the predictive value of sST2, sLR11, and hs-CRP for all-cause mortality and MACCEs, we evaluated the additive effects of each biomarker on the base model: model 1 , base model + sST2; model 2, base model + sLR11; model 3, base model + hs-CRP. The continuous net reclassification index (NRI), the integrated discrimination improvement (IDI) and $c$ Statistics were calculated to ascertain which biomarker improved prediction of the primary outcome when added to the base model. A $P$ value of less than 0.05 was considered to indicate statistical significance.

\section{Results}

Baseline characteristics of participants

The baseline characteristics of study participants are shown in Table 1 . The mean age of patients was $53.9 \pm$ 11.8 years, and 47 patients $(63.5 \%)$ were men. The median PD duration was 30.0 (16.0-96.0) months. A total of 21 patients $(28.4 \%)$ were diabetic and 10 patients (13.5\%) had a history of cardiovascular disease. Diabetic kidney disease was the most common cause of ESRD, followed by hypertensive nephrosclerosis and glomerulonephritis. The mean concentrations of serum sST2 and sLR11 were $75.0 \pm 26.6 \mathrm{ng} / \mathrm{mL}$ and $16.4 \pm 4.8 \mathrm{ng} / \mathrm{mL}$, respectively, the median value of hs-CRP was $0.96(0.62-$ 2.10) $\mathrm{mg} / \mathrm{L}$.

\section{Prognostic value of sST2 for all-cause mortality and MACCE}

During a median follow-up of 38.5 months, 13 (17.6\%) patients died and there were 23 (31.3\%) MACCEs. When patients were dichotomized based on the median value of sST2 $(70.9 \mathrm{ng} / \mathrm{mL})$, crude all-cause death rates were 1.74 and 10.18 per 100 person-years, and MACCE rates were 6.15 and 13.47 per 100 person-years in the low and high sST2 groups, respectively (Table S1). The cumulative survival rate was significantly lower in the high sST2 group (log-rank test; $P=0.002$ for all-cause mortality and $P=0.01$ for MACCEs) (Fig. 1). In multivariable Cox regression analysis, the high SST2 (above the median) was significantly associated with increased risk for allcause mortality and MACCEs (Table 2). When patients were categorized using calculated cut-off values of sST2, the high sST2 group had a 5.551- and 4.609-fold increased risk for all-cause mortality and MACCEs compared to the lower sST2 group. Moreover, the prognostic value of sST2 remained significant as a continuous variable (per $1 \mathrm{SD}$ increase, hazard ratio $[\mathrm{HR}]=$ 1.943; 95\% confidence interval $[\mathrm{CI}]=1.124-3.359$ for allcause mortality and $\mathrm{HR}=1.630 ; 95 \% \mathrm{CI}=1.073-2.477$ for MACCEs). When dialysis adequacy was added to multivariable models, the prognostic value of sST2 remained consistent (Table S2). Multivariable fractional polynomial analysis indicated that the risk of all-cause mortality and MACCEs increased steadily with higher sST2 levels, showing a linear relationship (Fig. 2).

\section{Prognostic value of sLR11 for all-cause mortality and MACCEs}

Patients were dichotomized based on the median value of sLR11 $(15.2 \mathrm{ng} / \mathrm{mL})$. All-cause death rates were 5.34 and 4.12 per 100 person-years, and MACCE rates were 9.78 and 7.76 per 100 person-years in the low and high sLR11 groups, respectively (Table S1). There were no differences in all-cause death or MACCE-free survival rates between the high and low sLR11 groups (both $P>$ 
Table 1 Baseline characteristics of study participants

\begin{tabular}{|c|c|}
\hline & $N=74$ \\
\hline Age (years) & $53.9 \pm 11.8$ \\
\hline Male, $n(\%)$ & $47(63.5)$ \\
\hline Duration of PD (months) & $30.0(16.0-96.0)$ \\
\hline \multicolumn{2}{|l|}{ Cases of ESRD, $n(\%)$} \\
\hline Diabetic kidney disease & $21(28.4 \%)$ \\
\hline Hypertensive nephrosclerosis & $19(25.7 \%)$ \\
\hline Glomerulonephritis & $17(23.0 \%)$ \\
\hline Polycystic kidney disease & $3(4.1 \%)$ \\
\hline${ }^{\mathrm{a}}$ Others & $3(4.1 \%)$ \\
\hline Unknown & $11(14.9 \%)$ \\
\hline Diabetes mellitus, n (\%) & $21(28.4)$ \\
\hline Cardiovascular disease, $n$ (\%) & $10(13.5)$ \\
\hline Ever-smokers, n (\%) & $29(39.2)$ \\
\hline Lipid-lowering therapy, n (\%) & $28(37.8)$ \\
\hline Systolic blood pressure (mmHg) & $133.7 \pm 21.5$ \\
\hline Diastolic blood pressure $(\mathrm{mmHg})$ & $75.0 \pm 11.6$ \\
\hline Body mass index $\left(\mathrm{kg} / \mathrm{m}^{2}\right)$ & $23.1 \pm 2.8$ \\
\hline Hemoglobin (g/L) & $104 \pm 11$ \\
\hline Glucose (mmol/L) & $5.3 \pm 2.4$ \\
\hline Blood urea nitrogen (mmol/L) & $7.4 \pm 2.1$ \\
\hline Creatinine $(\mu \mathrm{mol} / \mathrm{L})$ & $972.4 \pm 371.3$ \\
\hline Albumin $(g / L)$ & $35 \pm 5$ \\
\hline Triglyceride (mmol/L) & $1.3 \pm 0.8$ \\
\hline Total cholesterol (mmol/L) & $4.3 \pm 0.9$ \\
\hline LDL cholesterol (mmol/L) & $2.4 \pm 0.7$ \\
\hline HDL cholesterol (mmol/L) & $1.1 \pm 0.3$ \\
\hline Calcium (mmol/L) & $2.2 \pm 0.2$ \\
\hline Phosphorous (mmol/L) & $1.7 \pm 0.5$ \\
\hline Total Kt/N urea (per week) & $2.1 \pm 0.4$ \\
\hline nPCR (g/kg/day) & $0.97 \pm 0.17$ \\
\hline hs-CRP (mg/L) & $0.96(0.62-2.10)$ \\
\hline sST2 (ng/mL) & $75.0 \pm 26.6$ \\
\hline sLR11 (ng/mL) & $16.4 \pm 4.8$ \\
\hline
\end{tabular}

Data are expressed as mean \pm standard deviation, median (interquartile range), or number of patients (percent)

Abbreviations: hs-CRP high-sensitivity C-reactive protein, HDL high-density lipoprotein, $K t / N$ urea fractional urea clearance, $L D L$ low-density lipoprotein, $n P C R$ normalized protein catabolic rate, $P D$ peritoneal dialysis, $S L R 11$ soluble form of low-density lipoprotein receptor relative with 11 ligand-binding repeats, SST2 soluble form of suppression of tumorigenicity 2

${ }^{a}$ Others: interstitial nephritis, obstructive uropathy, or post status of nephrectomy

0.05) (Fig. 1). Multivariable Cox regression analysis showed there was no significant association between sLR11 and all-cause mortality or MACCEs, regardless of using median value $(15.2 \mathrm{ng} / \mathrm{mL})$ or calculated cut-off $(14.9 \mathrm{ng} / \mathrm{mL})$ (Table 2). Fractional polynomial analysis indicated a non-linear relationship (Fig. 2).
Predictive value of sST2, sLR11, and hs-CRP for all-cause mortality and MACCE

The predictive value of sST2, sLR11, and hs-CRP was evaluated using ROC analysis (Fig. 3), continuous NRI, IDI, and $c$ Statistics (Table 3). The AUCs of sST2 for allcause mortality and MACCEs were $0.699(P=0.03)$ and $0.644(P=0.04)$, respectively. In contrast, the AUC of sLR11 and hs-CRP were not significant. Furthermore, when SST2 was added to the base model that included traditional risk factors (model 1), only sST2 significantly improved the predictive ability of all-cause mortality (NRI $=0.598, \mathrm{P}=0.04)$. However, sLR11 (model 2) or hsCRP (model 3) did not increase predictability for allcause mortality. In terms of MACCE, there was no significant advantage from adding SST2, sLR11, or hs-CRP to the base model. Similar to IDI, the ability of discrimination was not significant in $c$ Statistics.

\section{Discussion}

In the present study, we first demonstrated that higher sST2 concentrations are independently associated with a greater risk of all-cause mortality and MACCEs in prevalent PD patients. In contrast, we did not find a significant association between sLR11 and study outcomes. Only sST2 improved the predictive ability of all-cause mortality, when added to the base model including traditional risk factors.

Previous studies indicated that ST2L, the transmembrane receptor form of ST2, exhibited cardio-protective effects associated with binding to interleukin-33 (IL-33), which results in a reduction in cardiomyocyte apoptosis and fibrosis or hypertrophy of injured cardiac tissues [4, 5]. The soluble form of ST2, sST2, is a decoy receptor $[6,7]$. As its concentration increases, sST2 competitively binds to IL-33 and disrupts the ST2L/IL-33 interaction, thus inhibiting cardio-protective signalling $[6,7]$. Therefore, the sST2 level has been widely investigated as a biomarker of cardiovascular disease, especially in patients with congestive heart failure [8-10]. Two recent meta-analyses demonstrate clearly that sST2 has a significant prognostic value in heart failure patients $[9,10]$. In chronic heart failure, sST2 also showed a significant prognostic value for all-cause and cardiovascular mortality [9]. A study of acute congestive heart failure found that higher sST2 concentrations at both admission and discharge are significantly associated with all-cause and cardiovascular mortality or hospitalization due to heart failure [10]. Furthermore, repeated measurements of sST2 concentration are useful for assessing the clinical course of heart failure, suggesting that sST2 is a suitable biomarker for disease monitoring as well as prognosis [27].

Only a few studies have investigated the prognostic value of sST2 in patients with kidney diseases. One study involving 883 pre-dialysis CKD patients showed that 

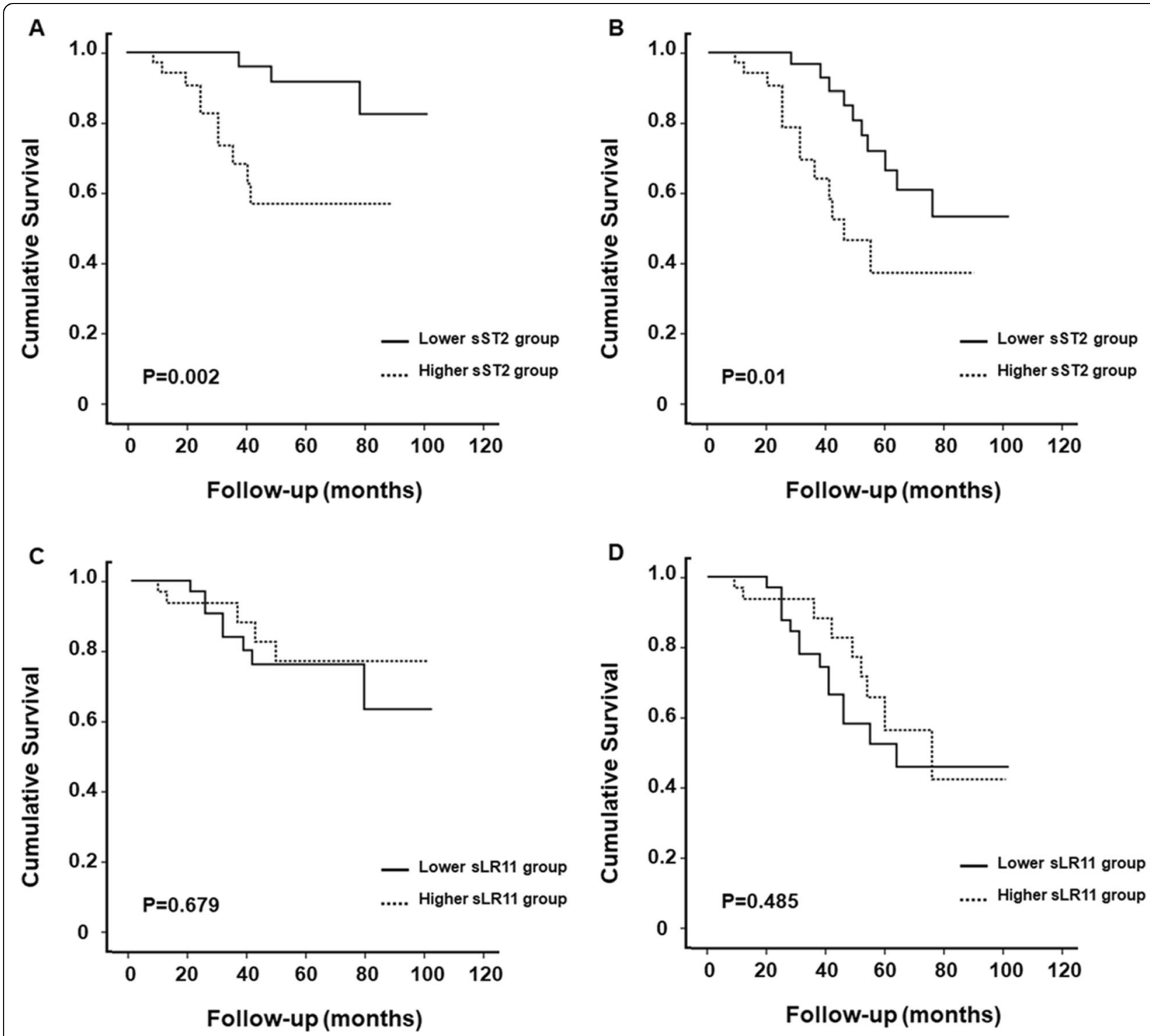

Fig. 1 Kaplan-Meier plots for study outcomes for high and low SST2 and SLR11 groups. The high sST2 group had significantly greater risk of allcause death (a) and MACCEs (b) compared with the low sST2 group. There were no significant differences in the risk of all-cause death (c) and MACCEs (d) between the high and low sLR11 groups. Abbreviations: MACCE, major adverse cardiac and cerebrovascular event; sLR11, soluble form of low-density lipoprotein receptor relative with 11 ligand-binding repeats; sST2, soluble form of suppression of tumorigenicity 2

higher sST2 concentrations are associated with increased mortality risk [25]. In a subgroup analysis of heart failure patients, high SST2 level remained a significant prognostic factor in patients with an estimated glomerular filtration rate $<60 \mathrm{~mL} / \mathrm{min} / 1.73 \mathrm{~m}^{2}$ [28]. In HD patients, two studies reported that $\mathrm{sST} 2$ had a significant association with all-cause mortality [23] and cardiovascular events [24]. In the current study, we revealed for the first time that prevalent PD patients with elevated SST2 levels have a greater risk of all-cause mortality and MACCEs. The significant prognostic value of sST2 remained unaltered when patients were categorized using both median value and calculated cut-off. To date, there is no definite sST2 cut-off for the prediction of clinical outcomes in populations with kidney diseases. Previous HD studies suggested a $48 \mathrm{ng} / \mathrm{mL}$ for all-cause mortality [23] or $58 \mathrm{ng} / \mathrm{mL}$ for MACCE [24], respectively. In this study, the calculated cut-off for sST2 was $75.8 \mathrm{ng} / \mathrm{mL}$ for all-cause mortality and $72.5 \mathrm{ng} / \mathrm{mL}$ for MACCE, which were higher than those of previous studies. In prevalent HD patients in Slovenia, the median value of sST2 was $28 \mathrm{ng} / \mathrm{mL}$ [23]. In a study of Korean incident HD patients, the median value of sST2 was $59.5 \mathrm{ng} / \mathrm{mL}$ [24]. Because our patients were prevalent PD patients with a median dialysis vintage of 30 months, we assumed that our patients may be at higher cardiovascular risk than incident dialysis patients, leading 
Table 2 Multivariable Cox proportional hazard models of sST2 and sLR11 for all-cause mortality and MACCEs

\begin{tabular}{|c|c|c|c|}
\hline & Crude HR (95\% Cl) & ${ }^{\mathrm{a} A d j u s t e d ~ H R ~} 1$ (95\% Cl) & ${ }^{\mathrm{b}}$ Adjusted HR 2 (95\% Cl) \\
\hline \multicolumn{4}{|l|}{ All-cause mortality } \\
\hline \multicolumn{4}{|l|}{ sST2 } \\
\hline${ }^{c} \mathrm{sST} 2 \geq 70.9 \mathrm{ng} / \mathrm{mL}$ (vs. < 70.9) & $6.715(1.749-25.784)$ & $9.638(2.180-42.602)$ & $10.144(2.156-47.734)$ \\
\hline dsST2 $\geq$ cut-off (vs. < cut-off) & $9.459(2.034-43.988)$ & $12.189(2.408-61.696)$ & $5.551(1.360-22.660)$ \\
\hline sST2 (per 1 SD increase) & $2.179(1.351-3.515)$ & $1.921(1.151-3.207)$ & $1.943(1.124-3.359)$ \\
\hline \multicolumn{4}{|l|}{ sLR11 } \\
\hline${ }^{c_{s L R}} 11 \geq 15.2 \mathrm{ng} / \mathrm{mL}(\mathrm{vs} .<15.2)$ & $0.791(0.258-2.422)$ & $0.630(0.187-2.130)$ & $0.601(0.168-2.145)$ \\
\hline${ }^{\mathrm{e}}$ sLR11 $\geq$ cut-off (vs. < cut-off) & $1.271(0.415-3.896)$ & $0.944(0.271-3.286)$ & $0.859(0.211-3.503)$ \\
\hline sLR11 (per 1 SD increase) & $0.884(0.503-1.555)$ & $0.763(0.397-1.467)$ & $0.719(0.356-1.451)$ \\
\hline \multicolumn{4}{|l|}{ MACCEs } \\
\hline \multicolumn{4}{|l|}{ sST2 } \\
\hline${ }^{c_{s S T} 2} \geq 70.9 \mathrm{ng} / \mathrm{mL}$ (vs. $\left.<70.9\right)$ & $2.056(1.259-6.940)$ & $4.623(1.743-12.263)$ & $3.928(1.413-10.918)$ \\
\hline $\mathrm{d}_{\mathrm{sST}} \mathrm{S} \geq$ cut-off (vs. <cut-off) & $3.776(1.546-9.218)$ & $5.445(2.020-14.679)$ & 4.609 (1.608-13.208) \\
\hline sST2 (per 1 SD increase) & $1.748(1.179-2.592)$ & $1.683(1.120-2.529)$ & $1.630(1.073-2.477)$ \\
\hline \multicolumn{4}{|l|}{ sLR11 } \\
\hline${ }^{c} \mathrm{sLR} 11 \geq 15.2 \mathrm{ng} / \mathrm{mL}(\mathrm{vs} .<15.2)$ & $0.743(0.321-1.723)$ & $0.736(0.29301 .849)$ & $0.737(0.271-2.002)$ \\
\hline${ }^{\mathrm{e}}$ sLR11 $\geq$ cut-off (vs. < cut-off) & $1.278(0.551-2.965)$ & $1.315(0.532-3.250)$ & $1.315(0.487-3.548)$ \\
\hline sLR11 (per 1 SD increase) & $0.951(0.634-1.428)$ & $0.947(0.606-1.481)$ & $0.927(0.577-1.490)$ \\
\hline \multicolumn{4}{|c|}{$\begin{array}{l}\text { Abbreviations: } C I \text { confidence interval, } H R \text { hazard ratio, MACCE major adverse cardiac and cerebrovascular event, PD peritoneal dialysis, SD standard deviation, sLR11 } \\
\text { soluble form of low-density lipoprotein receptor relative with } 11 \text { ligand-binding repeats, sST2 soluble form of suppression of tumorigenicity } 2 \\
\text { adjusted HR } 1 \text { was calculated after adjustment of age, sex, diabetes mellitus, and history of cardiovascular disease } \\
\text { b Adjusted HR } 2 \text { was calculated after adjustment of age, sex, PD duration, diabetes mellitus, history of cardiovascular disease, smoking status, body mass index, } \\
\text { and hemoglobin concentrations } \\
\text { cPatients were dichotomized based on median values of } s S T 2(70.9 \mathrm{ng} / \mathrm{mL}) \text { and sLR11 }(15.2 \mathrm{ng} / \mathrm{mL}) \text {, respectively } \\
\text { dPatients were categorized into two groups based on the calculated cut-off of sST2 for all-cause mortality }(75.8 \mathrm{ng} / \mathrm{mL}) \text { and MACCEs }(72.5 \mathrm{ng} / \mathrm{mL}) \\
\text { ePatients were categorized into two groups based on the calculated cut-off of sLR11 (14.9 ng/mL) for all-cause mortality and MACCEs }\end{array}$} \\
\hline
\end{tabular}

to higher cut-off and median value of sST2. However, we did not clarify the exact reason for the discrepancy in cutoff and median values of sST2, such as ethnicity, dialysis modality (HD or PD), or dialysis vintage. Future studies are needed to address this issue.

In the present study, the AUCs of sST2 for all-cause mortality and MACCE were significant and SST2 significantly improved the predictive ability for all-cause mortality when it was added to the base model that included traditional risk factors. From these results, we surmised that $\mathrm{SST} 2$ could be helpful in predicting adverse clinical outcomes in PD patients. However, the absolute values of the AUCs were not high (0.699 for all-cause mortality and 0.644 for MACCE) and SST2 did not show additive predictive value for MACCE compared with the base model. The discrimination index was not significant. These findings suggest that sST2 alone does not outweigh the predictive ability of traditional risk factors for cardiovascular outcomes. Nevertheless, fractional polynomial analyses found a linear association between sST2 concentrations and study outcome, which can be easily interpreted in clinical practice. Moreover, the sST2 concentration is independent of kidney function and CKD stage $[28,29]$ and not affected by haemodiafiltration procedures [30]. Interpretation of serum concentrations of biomarkers that are affected by kidney function or dialysis is complex and thus not easily applicable to realworld clinical settings. Based on these results, we speculate that sST2 can be a clinically relevant biomarker in PD patients. However, further investigation with a larger number of participants is necessary to evaluate the additive predictive value of sST2 for adverse cardiovascular outcomes in a PD population.

Previous studies have reported that sLR11 is implicated in coronary artery disease $[18,19]$, carotid atherosclerosis [20] and vascular calcification [21]. However, to date, sLR11 has not been investigated in patients with kidney diseases, and this study revealed that SLR11 concentration is not correlated with the risk of death or cardiovascular disease in prevalent PD patients. The mechanism by which LR11 mediates atherosclerosis reportedly involves the enhancement of vascular smooth muscle cell proliferation and migration [11-15]. LR11 produced by intimal smooth muscle cells induces increased smooth muscle cell migration in vitro via the upregulation of urokinase-type plasminogen activator receptor expression [15]. Of note, postprandial triglyceride-rich lipoproteins stimulate LR11 release from aortic smooth muscle cells, and 

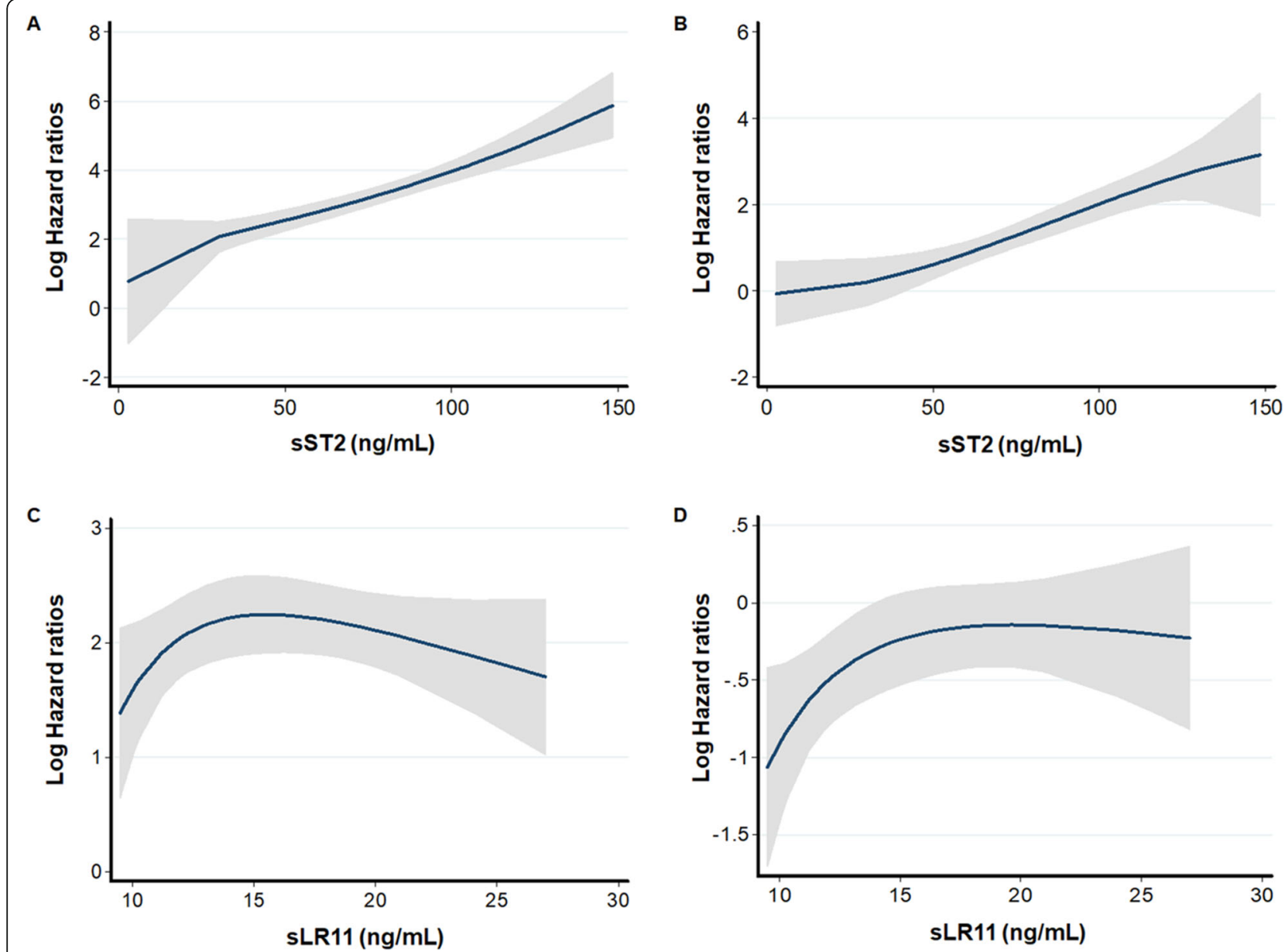

Fig. 2 Multivariable fractional polynomial graph for association of SST2 and SLR11 with study outcomes. Hazard ratios were calculated after adjustment for age, sex, peritoneal dialysis duration, diabetes mellitus, history of cardiovascular disease, smoking status, body mass index, and hemoglobin for all-cause death and MACCE. SST2 indicated a linear relationship with the risk of (a) all-cause mortality and (b) MACCEs. sLR11 did not show a remarkable trend with (c) all-cause mortality and (d) MACCEs. Shaded areas indicate $95 \%$ confidence intervals. Abbreviations: MACCE, major adverse cardiac and cerebrovascular event; sLR11, soluble form of low-density lipoprotein receptor relative with 11 ligand-binding repeats; sST2, soluble form of suppression of tumorigenicity 2

overexpression of LR11 increases binding of triglyceriderich lipoproteins but not low-density lipoprotein [31], suggesting a close association with triglycerides. In CKD patients, non-traditional risk factors, such as uraemia, inflammation, malnutrition or mineral-bone disease also contribute to a high risk of developing cardiovascular disease, in addition to the traditional risk factors such as advanced age, smoking, diabetes, and dyslipidaemia [32]. In this study, the mean triglyceride concentrations were 1.3 $\mathrm{mmol} / \mathrm{L}$, which is lower than the cut-off for hypertriglyceridemia $(>2.3 \mathrm{mmol} / \mathrm{L})$ in the general Korean population. Triglyceride level was not a significant risk factor for mortality or MACCEs in the univariate Cox analysis (data not shown). Considered collectively, these data suggest that the lower contribution of dyslipidaemia to the development of cardiovascular disease in a CKD setting could explain the non-significant association between SLR11 levels and prognosis in our patients. Furthermore, uraemia and impaired kidney function can alter the expression or metabolism of sLR11 in patients with kidney diseases, and dialysis procedures can also affect sLR11 concentrations. However, there are no available data on sLR11 concentrations in ESRD patients, thus further studies are needed to clarify this issue.

This study has several limitations. First, the number of study participants was small, resulting in a small number of primary outcomes. Although our multivariable models were well fitted (Table S3), the potential risk of overfitting still exists. In addition, we cannot exclude type II errors for the non-significant prognostic value of sLR11. To mitigate these confounding effects, a fractional polynomial analysis was performed to explore the association of sST2 and sLR11 with primary outcome using continuous terms. In the fractional polynomial 

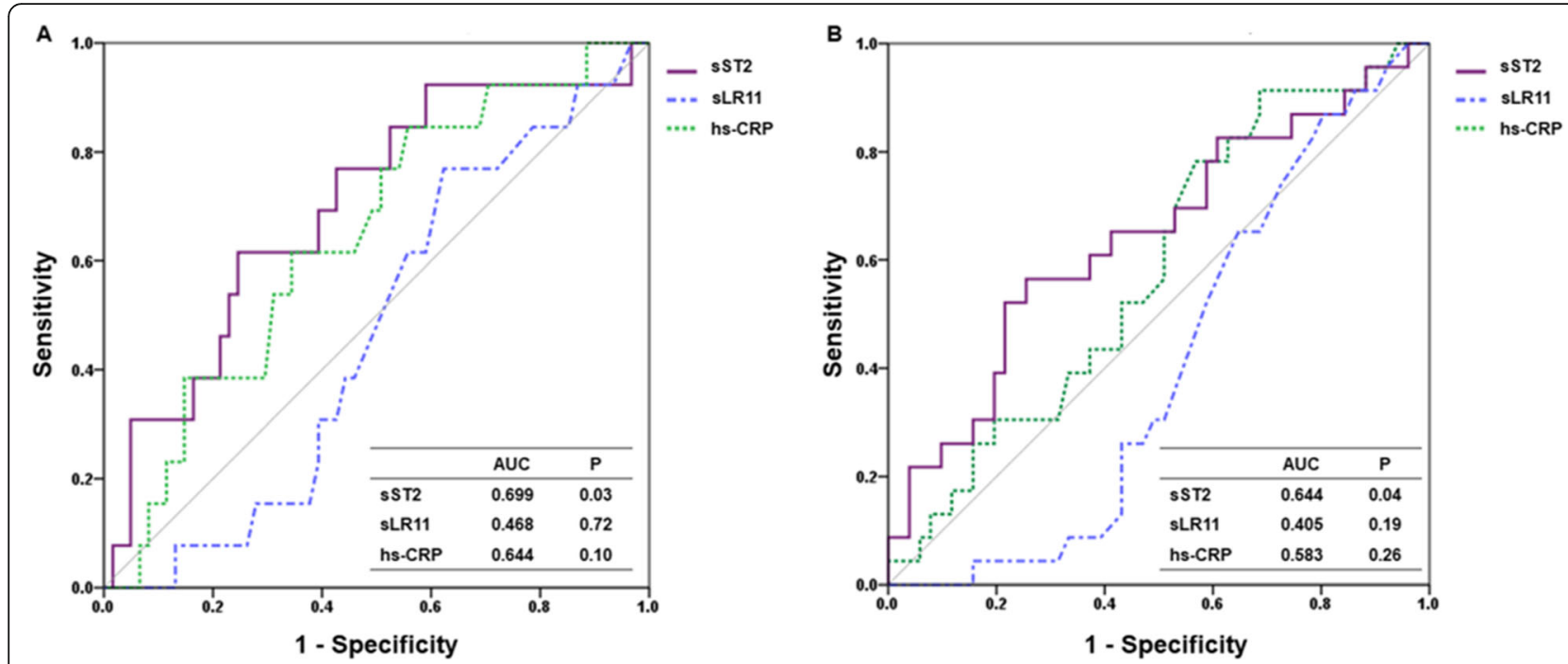

Fig. 3 Receiver operating characteristic curves for sST2, sLR11, and hs-CRP to predict all-cause mortality and MACCEs. sST2 exhibited a significant predictive value for (a) all-cause mortality and (b) MACCEs (both $P<0.05$ ). Abbreviations: hs-CRP, high-sensitivity C-reactive protein; MACCE, major adverse cardiac and cerebrovascular event; sLR11, soluble form of low-density lipoprotein receptor relative with 11 ligand-binding repeats; sST2, soluble form of suppression of tumorigenicity 2

analysis, sST2 showed a linear relationship but sLR11 showed a non-linear relationship with study outcomes. Therefore, even if our study was underpowered to detect a significant difference, the clinical utility of sLR11 as a biomarker is low due to the complexity of its interpretation, at least in PD patients. However, our findings should be interpreted with caution and should be confirmed by external validation in another independent cohort. Furthermore, a large-scale prospective investigation is mandatory to confirm our results. Second, this study analyzed only Korean prevalent PD patients. We have presumed that our participants were at high risk of cardiovascular disease due to a relatively long duration of PD. However, on the contrary, our participants have been tolerable to PD treatment until study enrolment. Given the high mortality risk in the early period of dialysis commencement, investigating prevalent PD patients may lead to a survival bias. Third, in the current study,

Table 3 NRIs, IDIs, and c Statistics for prediction of all-cause mortality and MACCE

\begin{tabular}{|c|c|c|c|c|c|c|}
\hline \multirow[t]{2}{*}{ Models } & NRI & ${ }^{a p}$ & $\mid \mathrm{IDI}$ & ap & c Statistics & ${ }^{a} \mathrm{p}$ \\
\hline & \multicolumn{2}{|l|}{$(95 \% \mathrm{Cl})$} & \multicolumn{4}{|l|}{$(95 \% \mathrm{Cl})$} \\
\hline \multicolumn{7}{|c|}{ All-cause mortality } \\
\hline Base model & Reference & & Reference & & 0.729 & \\
\hline Model 1 & 0.598 (0.039 to 1.156$)$ & 0.04 & $0.029(-0.0068$ to 0.0655$)$ & 0.11 & 0.863 & 0.48 \\
\hline Model 2 & $0.225(-0.372$ to 0.821$)$ & 0.46 & $0.005(-0.014$ to 0.024$)$ & 0.61 & 0.731 & 0.19 \\
\hline Model 3 & $0.477(-0.106$ to 1.059$)$ & 0.11 & $0.027(-0.0005$ to 0.060$)$ & 0.10 & 0.765 & 0.71 \\
\hline \multicolumn{7}{|l|}{ MACCE } \\
\hline Base model & Reference & & Reference & & 0.718 & \\
\hline Model 1 & $0.385(-0.099$ to 0.870$)$ & 0.12 & $0.008(-0.014$ to 0.030$)$ & 0.49 & 0.801 & 0.46 \\
\hline Model 2 & $0.133(-0.357$ to 0.627$)$ & 0.59 & $0.019(-0.013$ to 0.050$)$ & 0.24 & 0.705 & 0.33 \\
\hline Model 3 & $0.007(-0.481$ to 0.495$)$ & 0.9 & $0.001(-0.002$ to 0.007$)$ & 0.82 & 0.720 & 0.47 \\
\hline
\end{tabular}

Base model: adjusted for age, sex, PD duration, smoking, diabetes mellitus, cardiovascular disease, body mass index, and hemoglobin

Model 1: base model $+\mathrm{sST} 2$

Model 2: base model + sLR11

Model 3: base model + hs-CRP

Abbreviations: $C l$ confidence interval, IDI integrated discrimination improvement, $h s-C R P$ high-sensitivity C-reactive protein, $P D$ peritoneal dialysis, MACCE major adverse cardiac and cerebrovascular event, NRI net reclassification index, sLR11 soluble form of low-density lipoprotein receptor relative with 11 ligand-binding repeats, $S$ ST2 soluble form of suppression of tumorigenicity 2

${ }^{\mathrm{a}} \mathrm{v}$. Base model 
the proportion of participants who had diabetes mellitus was $28.4 \%$, which is lower than the $50.2 \%$ of Korean dialysis patients [33]. Moreover, only $13.5 \%$ of the participants had a history of cardiovascular disease. These findings suggest that our study participants were more likely to be in a better clinical condition compared with other PD cohorts, introducing a selection bias. Furthermore, several patients were excluded from the original cohort due to lack of blood samples or withdrawal from further study participation, leading to the potential for another selection bias. To abrogate the effect of selection bias, we analyzed differences in baseline characteristics between patients with and without biomarker measurements. We found no significant differences in baseline characteristics except for PD duration and haemoglobin between patients who had available sST2 and sLR11 concentration measurements and those who did not (Table S4). Nevertheless, 74 patients may not be representative of all participants in our original cohort. Fourth, this study included a small number of participants from a single tertiary hospital in Korea. Because there are huge differences in medical resources and dialysis practice pattern among institutions, our results may not be generalized to other populations. Fifth, due to the observational nature of this study, we cannot evaluate the causal relationship between sST2 or sLR11 and the development of our primary outcome. Although we surmised that a significant association of SST2 with cardiac fibrosis, myocardial hypertrophy, or ventricular dysfunction observed in previous studies may contribute to the prognostic value of sST2 on mortality and MACCEs, underlying mechanisms were not investigated in this study. Furthermore, the effects of changes in SST2 and sLR11 on mortality or cardiovascular disease were not evaluated, exerting an unmeasured confounding effect. Although multivariable Cox models were constructed with significant variables from univariate analysis and additional variables which were known to be associated with adverse clinical outcome in PD patients, we cannot totally exclude residual confounding effects derived from the observational study. Sixth, because there is no definite cut-off for sST2 and sLR11 in patients with kidney diseases, we calculated the cut-off value using ROC analysis. These cut-offs need to be validated in further studies. Lastly, we only determined concentrations of sST2 and sLR11 and did not evaluate other emerging cardiovascular biomarkers, such as growth differentiation factor 15 or galectin 3. Determining and comparing the prognostic value of various new biomarkers is worthwhile. Despite these limitations, we believe that the current study provides useful information regarding the prognostic value of emerging cardiovascular biomarkers in $\mathrm{PD}$ patients.

\section{Conclusions}

The present study reveals that sST2 is a significant prognostic factor in PD patients. However, we found no significant association between SLR11 and the study outcomes. These findings suggest that sST2 may be a useful biomarker for stratifying mortality and cardiovascular risk in patients undergoing PD. However, additional studies to validate these emerging cardiovascular biomarkers in this population are needed.

\section{Supplementary information}

Supplementary information accompanies this paper at https://doi.org/10. 1186/s12882-020-01886-7.

Additional file 1: Table S1. All-cause death and MACCE according to the sST2 and SLR11 groups. Table S2. Multivariable Cox proportional hazard models of SST2 and SLR11 for all-cause mortality and MACCEs with adjustment of dialysis adequacy. Table S3. Hosmer-Lemeshow test for calibration of models. Table S4. Baseline characteristics of patients with and without SST2 and SLR11 values.

\section{Abbreviations}

CKD: Chronic kidney disease; ESRD: End-stage renal disease;

HD: Hemodialysis; hs-CRP: High-sensitivity C-reactive protein; IDI: Integrated discrimination improvement; MACCE: Major adverse cardiac and

cerebrovascular event; NRI: Net reclassification index; PD: Peritoneal dialysis; SLR11: Soluble form of low-density lipoprotein receptor relative with 11 ligand-binding repeats; sST2: Soluble form of suppression of tumorigenicity 2

\section{Acknowledgements}

Not applicable.

\section{Authors' contributions}

YBC, MJL, T-HY and HJK designed and conceptualized the study; MJL, JTP, SHH conducted the experiments; YBC and MJL analyzed the data; JTP, SHH, S-WK, T-HY, and HJK supervised the experiments and analyses; YBC, MJL, THY, and HJK wrote the manuscript; all authors critically analyzed the manuscript and approved the final version of the manuscript.

Authors' information

Not applicable.

\section{Funding}

This research was supported by Basic Science Research Program through the National Research Foundation of Korea (NRF) funded by the Ministry of Science, ICT \& Future Planning (NRF-2019R1C1C1010552). The funders of this study had no role in study design, data collection and analysis, decision to publish, or preparation of the manuscript.

Availability of data and materials

The datasets used for the current study are available from the corresponding author on reasonable request.

\section{Ethics approval and consent to participate}

The study was carried out in accordance with the Declaration of Helsinki and approved by the Institutional Review Board of the YUHS Clinical Trial Center (IRB No. 4-2016-0932). We obtained informed written consent from all participants.

Consent for publication

Not applicable.

Competing interests

The authors have no conflicts of interest to declare. 


\section{Author details}

${ }^{1}$ Department of Internal Medicine, CHA Bundang Medical Center, CHA University, 59 Yatap-ro, Bundang-gu, Seongnam-si, Korea. ${ }^{2}$ Department of Medicine, Graduate School of Yonsei University College of Medicine, Seoul, Korea. ${ }^{3}$ Department of Internal Medicine, College of Medicine, Institute of Kidney Disease Research, Yonsei University, Seoul, Korea.

Received: 1 October 2019 Accepted: 5 June 2020

Published online: 15 June 2020

\section{References}

1. McAlister FA, Ezekowitz J, Tonelli M, Armstrong PW. Renal insufficiency and heart failure: prognostic and therapeutic implications from a prospective cohort study. Circulation. 2004;109(8):1004-9.

2. House AA, Anand I, Bellomo R, Cruz D, Bobek I, Anker SD, Aspromonte N, Bagshaw S, Berl T, Daliento L, et al. Definition and classification of cardiorenal syndromes: workgroup statements from the 7th $A D Q I$ consensus conference. Nephro Dial Transplant. 2010;25(5):1416-20.

3. Pecoits-Filho R, Barberato SH. Echocardiography in chronic kidney disease: diagnostic and prognostic implications. Nephron Clin Pract. 2010;114(4): c242-7.

4. Weinberg EO, Shimpo M, De Keulenaer GW, MacGillivray C, Tominaga S, Solomon SD, Rouleau JL, Lee RT. Expression and regulation of ST2, an interleukin-1 receptor family member, in cardiomyocytes and myocardial infarction. Circulation. 2002;106(23):2961-6.

5. Seki K, Sanada S, Kudinova AY, Steinhauser ML, Handa V, Gannon J, Lee RT. Interleukin-33 prevents apoptosis and improves survival after experimental myocardial infarction through ST2 signaling. Circ Heart Fail. 2009;2(6):684-91.

6. Kakkar R, Lee RT. The IL-33/ST2 pathway: therapeutic target and novel biomarker. Nat Rev Drug Discov. 2008;7(10):827-40.

7. Daniels LB, Bayes-Genis A. Using ST2 in cardiovascular patients: a review. Futur Cardiol. 2014;10(4):525-39.

8. Weir RA, Miller AM, Murphy GE, Clements S, Steedman T, Connell JM, McInnes IB, Dargie HJ, McMurray JJ. Serum soluble ST2: a potential novel mediator in left ventricular and infarct remodeling after acute myocardial infarction. J Am Coll Cardiol. 2010;55(3):243-50.

9. Aimo A, Vergaro G, Passino C, Ripoli A, Ky B, Miller WL, Bayes-Genis A, Anand I, Januzzi IL, Emdin M. Prognostic value of soluble suppression of Tumorigenicity-2 in chronic heart failure: a meta-analysis. JACC Heart Fail. 2017;5(4):280-6.

10. Aimo A, Vergaro G, Ripoli A, Bayes-Genis A, Pascual Figal DA, de Boer RA, Lassus J, Mebazaa A, Gayat E, Breidthardt T, et al. Meta-analysis of soluble suppression of Tumorigenicity-2 and prognosis in acute heart failure. JACC Heart Fail. 2017;5(4):287-96.

11. Yamazaki H, Bujo H, Kusunoki J, Seimiya K, Kanaki T, Morisaki N, Schneider WJ, Saito Y. Elements of neural adhesion molecules and a yeast vacuolar protein sorting receptor are present in a novel mammalian low density lipoprotein receptor family member. J Biol Chem. 1996;271(40):24761-8.

12. Jacobsen L, Madsen P, Moestrup SK, Lund AH, Tommerup N, Nykjaer A, Sottrup-Jensen L, Gliemann J, Petersen CM. Molecular characterization of a novel human hybrid-type receptor that binds the alpha2-macroglobulin receptor-associated protein. J Biol Chem. 1996;271(49):31379-83.

13. Kanaki T, Bujo H, Hirayama S, Ishii I, Morisaki N, Schneider WJ, Saito Y. Expression of $L R 11$, a mosaic $L D L$ receptor family member, is markedly increased in atherosclerotic lesions. Arterioscler Thromb Vasc Biol. 1999; 19(11):2687-95.

14. Zhu Y, Bujo H, Yamazaki H, Hirayama S, Kanaki T, Takahashi K, Shibasaki M, Schneider WJ, Saito Y. Enhanced expression of the LDL receptor family member LR11 increases migration of smooth muscle cells in vitro. Circulation. 2002;105(15):1830-6.

15. Jiang M, Bujo H, Ohwaki K, Unoki H, Yamazaki H, Kanaki T, Shibasaki M, Azuma K, Harigaya K, Schneider WJ, et al. Ang II-stimulated migration of vascular smooth muscle cells is dependent on LR11 in mice. J Clin Invest. 2008;118(8):2733-46.

16. Takahashi M, Bujo H, Jiang M, Noike H, Saito Y, Shirai K. Enhanced circulating soluble LR11 in patients with coronary organic stenosis. Atherosclerosis. 2010;210(2):581-4.

17. Nishii K, Nakaseko C, Jiang M, Shimizu N, Takeuchi M, Schneider WJ, Bujo H. The soluble form of $L R 11$ protein is a regulator of hypoxia-induced, urokinase-type plasminogen activator receptor (UPAR)-mediated adhesion of immature hematological cells. J Biol Chem. 2013;288(17):11877-86.
18. Ogita M, Miyauchi K, Dohi T, Tsuboi S, Miyazaki T, Yokoyama T, Yokoyama K, Shimada K, Kurata T, Jiang M, et al. Increased circulating soluble LR11 in patients with acute coronary syndrome. Clin Chim Acta. 2013:415:191-4.

19. Ogita M, Miyauchi K, Jiang M, Kasai T, Tsuboi S, Naito R, Konishi H, Dohi T, Yokoyama T, Okazaki S, et al. Circulating soluble LR11, a novel marker of smooth muscle cell proliferation, is enhanced after coronary stenting in response to vascular injury. Atherosclerosis. 2014;237(1):374-8.

20. Jin W, Jiang $M, \operatorname{Han} X$, Han $X$, Murano T, Hiruta N, Ebinuma H, Piao L, Schneider WJ, Bujo H. Circulating soluble form of LR11, a regulator of smooth muscle cell migration, is a novel marker for intima-media thickness of carotid arteries in type 2 diabetes. Clin Chim Acta. 2016;457:137-41.

21. Vongpromek R, Bos S, Ten Kate GR, Bujo H, Jiang M, Nieman K, Schneider W, Roeters van Lennep JE, AJM V, EJG S, et al. Soluble LR11 associates with aortic root calcification in asymptomatic treated male patients with familial hypercholesterolemia. Atherosclerosis. 2017;265:299-304.

22. Gungor O, Unal HU, Guclu A, Gezer M, Eyileten T, Guzel FB, Altunoren O, Erken E, Oguz Y, Kocyigit I, et al. IL-33 and ST2 levels in chronic kidney disease: associations with inflammation, vascular abnormalities, cardiovascular events, and survival. PLoS One. 2017;12(6):e0178939.

23. Homsak E, Ekart R. ST2 as a novel prognostic marker in end-stage renal disease patients on hemodiafiltration. Clin Chim Acta. 2018;477:105-12.

24. Seo SM, Kim SH, Kim Y, Yoon HE, Shin SJ. Prognostic utility of soluble suppression of Tumorigenicity 2 level as a predictor of clinical outcomes in incident hemodialysis patients. Int J Med Sci. 2018;15(7):730-7.

25. Tuegel C, Katz R, Alam M, Bhat Z, Bellovich K, de Boer I, Brosius F, Gadegbeku C, Gipson D, Hawkins J, et al. GDF-15, galectin 3, soluble ST2, and risk of mortality and cardiovascular events in CKD. Am J Kidney Dis. 2018;72(4):519-28

26. Lee MJ, Lee SA, Nam BY, Park S, Lee SH, Ryu HJ, Kwon YE, Kim YL, Park KS, Oh HJ, et al. Irisin, a novel myokine is an independent predictor for sarcopenia and carotid atherosclerosis in dialysis patients. Atherosclerosis. 2015;242(2):476-82.

27. Maisel AS, Richards AM, Pascual-Figal D, Mueller C. Serial ST2 testing in hospitalized patients with acute heart failure. Am J Cardiol. 2015;115(7 Suppl):32B-7B.

28. Bayes-Genis A, Zamora E, de Antonio M, Galan A, Vila J, Urrutia A, Diez C, Coll R, Altimir S, Lupon J. Soluble ST2 serum concentration and renal function in heart failure. J Cardiac Fail. 2013;19(11):768-75.

29. De Berardinis B, Gaggin HK, Magrini L, Belcher A, Zancla B, Femia A, Simon M, Motiwala S, Bhardwaj A, Parry BA, et al. Comparison between admission natriuretic peptides, NGAL and SST2 testing for the prediction of worsening renal function in patients with acutely decompensated heart failure. Clin Ehcm Lab Med. 2015;53(4):613-21.

30. Homsak E, Ekart R. Hemodiafiltration affects NT-proBNP but not ST2 serum concentration in end-stage renal disease patients. Clin Biochem. 2016; 49(15):1159-63.

31. Vongpromek R, Bujo H, Hoekstra M, Schneider WJ, van der Zee L, Schinkel AF, Korporaal SJ, Dik WA, Ebinuma H, Jiang M, et al. LR11/SorLA links triglyceride-rich lipoproteins to risk of developing cardiovascular disease in FH patients. Atherosclerosis. 2015;243(2):429-37.

32. Chen J, Mohler ER, Xie D, Shlipak M, Townsend RR, Appel L, Ojo A, Schreiber M, Nessel L, Zhang $X$, et al. Traditional and non-traditional risk factors for incident peripheral arterial disease among patients with chronic kidney disease. Nehprol Dial Transplant. 2016;31(7):1145-51.

33. Jin DC, Yun S, Lee SW, Han S, Kim W, Park J, Kim Y. Current characteristics of dialysis therapy in Korea: 2016 registry data focusing on diabetic patients. Kidney Res Clin Pract. 2018;37:20-9.

\section{Publisher's Note}

Springer Nature remains neutral with regard to jurisdictional claims in published maps and institutional affiliations. 